\title{
CONHECIMENTO SOBRE NUTRIÇÃO DE PRATICANTES DE EXERCíCIO FÍSICO DE UMA ACADEMIA DE GINÁSTICA DE CRICIÚMA-SC
}

\section{Knowledge on nutrition of physical exercise practitioners of a fitness center at Criciúma-SC}

Pâmela Colonetti Arns ${ }^{1}$, Ângela Martinha Bongiolo¹, Maria Cristina Gonçalves de Souza $^{1}$, Rita Suselaine Vieira Ribeiro ${ }^{1}$, Ingrid Dalira Schweigert Perry ${ }^{1,2}$, Marco Antonio da Silva ${ }^{1}$

1 Departamento de Nutrição, Unidade Acadêmica da Saúde, Universidade do Extremo Sul Catarinense - UNESC, Criciúma, SC.

2 Programa de Pós Graduação em Saúde Coletiva (Mestrado Profissional), Unidade Acadêmica da Saúde - UNESC, Criciúma, SC.

\section{Endereço para correspondência:}

Dr. Marco Antonio da Silva

Universidade do Extremo Sul Catarinense

Bairro Universitário, Criciúma - SC

Fone: +55 48 3431-2560 Fax: +55 48 3431-2736

CEP: 88806-000

Email:mar@unesc.net 


\section{Resumo}

O objetivo do estudo foi avaliar o conhecimento sobre nutrição dos praticantes de exercício físico de uma academia de ginástica de Criciúma-SC. Participaram do estudo 163 praticantes, sendo 58 do sexo feminino e 105 do sexo masculino. Para analisar o conhecimento nutricional, o instrumento utilizado na coleta de dados foi o questionário desenvolvido para triatletas de Bassit e Malverdi (1998). Para analisar o perfil socioeconômico dos participantes foi utilizado o questionário ABEP 2015 e para avaliar o estado nutricional, foi calculado o Índice de Massa Corporal (IMC) dos praticantes (WHO, 1995). A análise dos dados foi realizada por meio da estatística descritiva (frequência e percentual) e inferencial (teste qui-quadrado). Os resultados revelaram, de modo geral, que o conhecimento nutricional dos praticantes de exercícios físicos se apresentou moderado. Além disso, destaca-se que foram constatadas diferenças estatísticas significativas entre o conhecimento nutricional e as variáveis frequência da prática e tempo de prática de exercícios físicos. Os praticantes de exercício físico possuem maior dificuldade em saber o valor energético dos nutrientes, bem como aos nutrientes que mais devem ser consumidos na dieta, porém, possuem maior conhecimento sobre função dos macronutrientes, seus alimentos-fonte e suas substituições.

Palavras-chave: Conhecimento; Nutrição; Exercício Físico.

\section{Abstract}

The aim of this study was to evaluate the knowledge of nutrition of exercise practitioners of a fitness center at Criciuma-SC. The study included 163 practitioners, 58 female and 105 male. To analyze the nutritional knowledge, the instrument used for data collection was the questionnaire developed for triathletes by Bassit and Malverdi (1998). To analyze the socioeconomic profile of the participants was used ABEP 2015 questionnaire and to assess the nutritional status, it calculated the body mass index (BMI) of practitioners (WHO, 1995). Data analysis was performed using descriptive statistics (frequency and percentage) and inferential (chi-square test). The results showed, in general, the nutritional knowledge of practitioners of physical exercises performed moderate. In addition, it is emphasized that statistically significant differences were found between nutritional knowledge and variable 


\section{Artigo Original \\ Exercício na Saúde, na doença e no esportivo}

frequency of practice and time for physical exercise. The exercise of practitioners have more difficulty in knowing the energy value of nutrients, and the nutrients that more should be consumed in the diet, however, have greater knowledge about function of macronutrients, their food sources and their replacements.

Keywords: Knowledge; Nutrition; Physical Exercise.

\section{INTRODUÇÃO}

A alimentação é um componente fundamental para uma boa qualidade de vida, pois além do prazer que proporciona, os alimentos são essenciais para a vida. Todos precisam se alimentar e isso deve ser feito de maneira balanceada e diversificada, levando-se em consideração que a quantidade e a diversidade dos alimentos ingeridos influenciam na obtenção dos nutrientes necessários ao organismo. Cada indivíduo possui seus próprios gostos, preferências e hábitos alimentares, contudo, são necessários cuidados como: a manutenção do peso corporal, o combate à obesidade, a eliminação do cigarro, a diminuição do consumo exagerado de álcool e do consumo de grandes quantidades de sal, gordura e açúcar refinado. Além disso, é preciso que haja a pratica de esportes ${ }^{1}$.

O exercício físico está se tornando um meio cada vez mais importante de prevenir e tratar muitas das doenças crônicas (cardiopatias coronarianas, acidente vascular cerebral, hipertensão, câncer, diabetes, artrite, osteoporose, doenças pulmonares crônicas e obesidade) $)^{2}$.

Tanto o exercício adequado como os bons hábitos alimentares podem proporcionar separadamente benefícios à saúde, mas a redução do total de fatores de risco é maximizada quando os dois (nutrição e exercícios adequados), fazem parte de um estilo de vida saudável².

A nutrição constitui o alicerce para o desempenho físico, de modo que proporciona o combustível para o trabalho biológico e as substancias químicas para extrair e utilizar a energia potencial dos alimentos ${ }^{3}$, sendo que as necessidades nutricionais de atletas e praticantes de atividades físicas são aumentadas em relação aos não praticantes. Se estes praticantes possuírem uma alimentação equilibrada, balanceada e variada e tiverem condições de ingerir todos os alimentos 


\section{Artigo Original \\ Exercício na Saúde, na doença e no esportivo}

necessários em quantidades e qualidade adequadas de acordo com a demanda física, suas necessidades podem ser supridas por meio apenas dos alimentos ${ }^{4}$.

Nas academias, o público, em sua maioria, é composto, por praticantes de atividade física e deve-se levar em consideração suas peculiaridades, como: hábitos regionais, classe socioeconômica, crenças e recursos teóricos (de informação) e práticos (disponibilidade de alimentos e produtos). Cabe ao profissional nutricionista traçar o perfil de seu público no dia-a-dia, pois irá esbarrar com crenças do próprio cliente, que quer a todo custo fazer uma dieta muito restrita ou hiperproteica, evitar o consumo de gorduras de maneira exagerada ou até mesmo insistir no consumo de suplementos e produtos para atletas ${ }^{5}$. Para que se obtenham práticas alimentares saudáveis e um maior rendimento, um conhecimento nutricional satisfatório torna-se importante ${ }^{6}$.

Diante do exposto, o presente estudo tem por objetivo avaliar 0 conhecimento sobre nutrição dos praticantes de exercício físico de uma academia de ginástica de Criciúma-SC.

\section{MÉTODOS}

A presente pesquisa se caracteriza como um estudo descritivo, com abordagem quantitativa de temporalidade transversal, com amostragem não probabilística e captação por conveniência.

A amostra deste estudo foi constituída por 163 indivíduos adultos, de ambos os sexos, com idade entre 20 a 59 anos, que frequentavam regularmente a academia de ginástica. Os critérios de inclusão adotados foram: estar matriculado na academia parceira da pesquisa, ter idade entre 20 e 59 anos e aceitar participar da pesquisa, assinando o Termo de Consentimento Livre e Esclarecido.

Para investigar o conhecimento nutricional, foi utilizado um questionário desenvolvido para triatletas, por Bassit e Malverdi ${ }^{7}$. O questionário contém 10 questões que abrangem as funções dos macronutrientes no organismo humano, principais fontes alimentares, distribuição normal (\%) dos macronutrientes, os grupos de substitutos alimentares, a diferença entre nutriente e alimento, a relação entre os nutrientes, o fornecimento de energia e se já houve orientação por um nutricionista. Para análise dos dados coletados, foram consideradas como corretas as questões 


\section{Artigo Original \\ Exercício na Saúde, na doença e no esportivo}

com mais de $75 \%$ de acertos, como parcialmente corretas as com 50 a $75 \%$ e como incorretas as com menos de $50 \%$ de acertos. Para cada questão correta foi atribuído o valor 1,0, para parcialmente correta o valor 0,5 e para incorreta não foi atribuído valor $(0,0)$. Posteriormente foram criados pontos de corte a partir da somatória de acertos das 9 questões do questionário, sendo de 1,0 a 3,5: nível de conhecimento baixo, de 4,0 a 6,5: nível de conhecimento moderado e de 7 a 9: nível de conhecimento alto.

O perfil socioeconômico foi obtido através do questionário ABEP 2015\%; o estado nutricional obtido através do Índice de Massa Corporal (IMC), sendo que o peso e a altura foram auto referidos pelos participantes. Quando o participante não soube mencioná-los, os valores foram obtidos pela entrevistadora no próprio estabelecimento de realização da pesquisa, através do uso de uma balança profissional mecânica antropométrica com estadiômetro da marca Balmak 111. A classificação do estado nutricional seguiu os valores preconizados pela Organização Mundial da Saúde (OMS) ${ }^{9}$.

O tratamento estatístico foi realizado por meio das análises descritiva (frequência e percentual) e inferencial (teste do qui-quadrado de Pearson), através do software Statistical Package for the Social Sciences (SPSS), versão 20, desenvolvido pela Internacional Business Machines ${ }^{\circledR}$ (IBM) para Microsoft Windows $\mathbb{B}$.

Este trabalho atendeu as premissas da Resolução CNS 466/12 e foi submetido e aprovado pelo Comitê de Ética em Pesquisa da UNESC sob o parecer no 1.348 .542 .

\section{RESULTADOS}

Na presente pesquisa, $64,42 \%$ dos participantes foram do sexo masculino $(n=105)$ e $35,58 \%$ do sexo feminino $(n=58)$, totalizando 163 pessoas.

Entre os participantes da pesquisa, segundo o critério da $\mathrm{ABEP}^{8}$, a maioria pertence à classe socioeconômica $B 2$, com 42,94\% $(n=70)$, seguida da B1 e $C 1$, $20,86 \%$ ( $n=34)$ e 19,63\% ( $n=32)$, respectivamente.

Com relação ao tempo de prática de exercícios físicos, 32\% (n=52) dos 162 indivíduos que responderam à pergunta afirmaram que se exercitam há quatro anos 


\section{Artigo Original \\ Exercício na Saúde, na doença e no esportivo}

ou mais e $28 \%(n=46)$ iniciaram a prática a menos de seis meses, com frequência da prática $(n=153)$ em quatro vezes $(20 \%)$ ou mais (39\%) por semana.

Ao analisar o IMC dos participantes da pesquisa que informaram seu peso e altura $(n=152)$, pode-se observar que a maioria se encontra eutrófico $(54,61 \%)$, embora o sobrepeso e a obesidade perfaçam $42,1 \%$ e $3,29 \%$ com baixo peso (Figura 1).

Figura 1. Classificação do estado nutricional segundo o Índice de Massa Corporal dos praticantes de exercícios físicos de uma academia de Criciúma-SC.

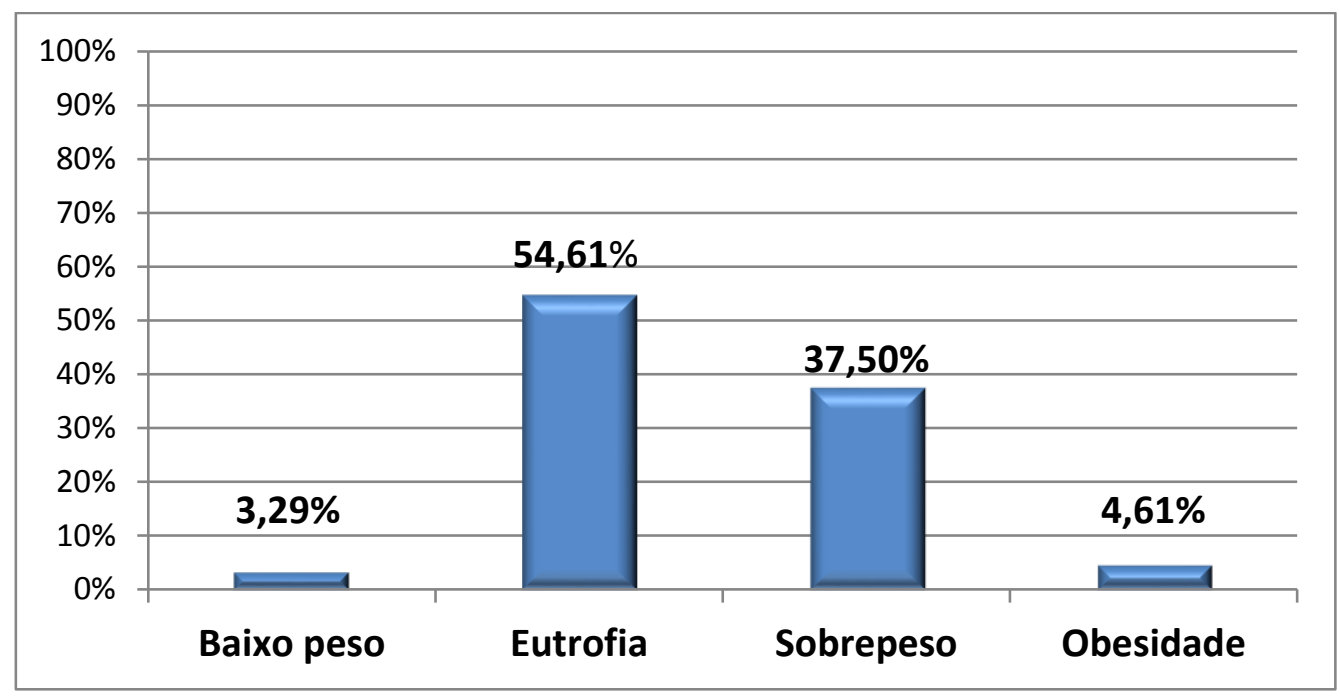

Do total dos participantes da pesquisa, 73,13\% de 160 praticantes de exercícios físicos afirmaram não terem sido orientados por nutricionista.

O conhecimento sobre nutrição de praticantes de exercícios físicos pode ser observado na Figura 2. De modo geral, percebe-se que a maioria dos praticantes $(66,25 \%)$ apresentou nível moderado de conhecimentos básicos sobre nutrição, seguido do nível baixo (22,69\%).

A Figura 3 apresenta os erros e acertos relacionados a cada questão do questionário de conhecimentos básicos sobre nutrição. Pode-se observar grande frequência de erros nas questões 2, 3 e 9 (mais de 50\% de erros), sendo que as que apresentaram mais acertos foram as de número 1, 6, 7 e 8 (com mais de $50 \%$ de acertos). 
Figura 2. Distribuição do percentual de praticantes de exercícios físicos de uma academia de Criciúma-SC segundo o nível de conhecimentos em nutrição.

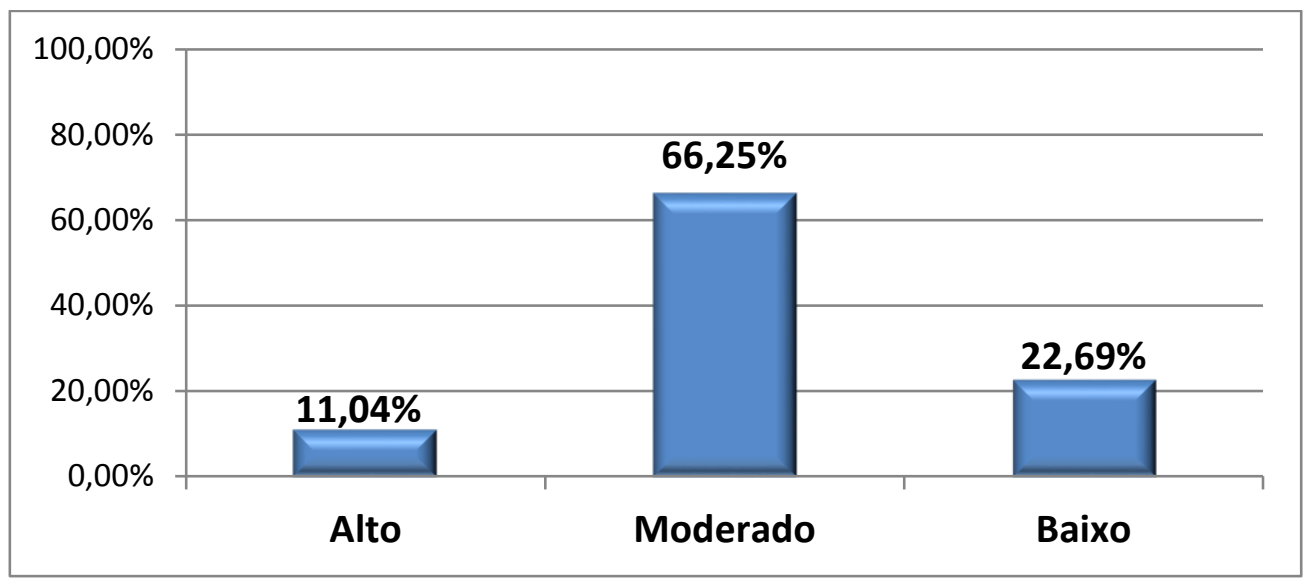

Figura 3. Porcentagem de acertos por questão do questionário de conhecimentos em nutrição de praticantes de exercícios físicos de uma academia de Criciúma-SC.

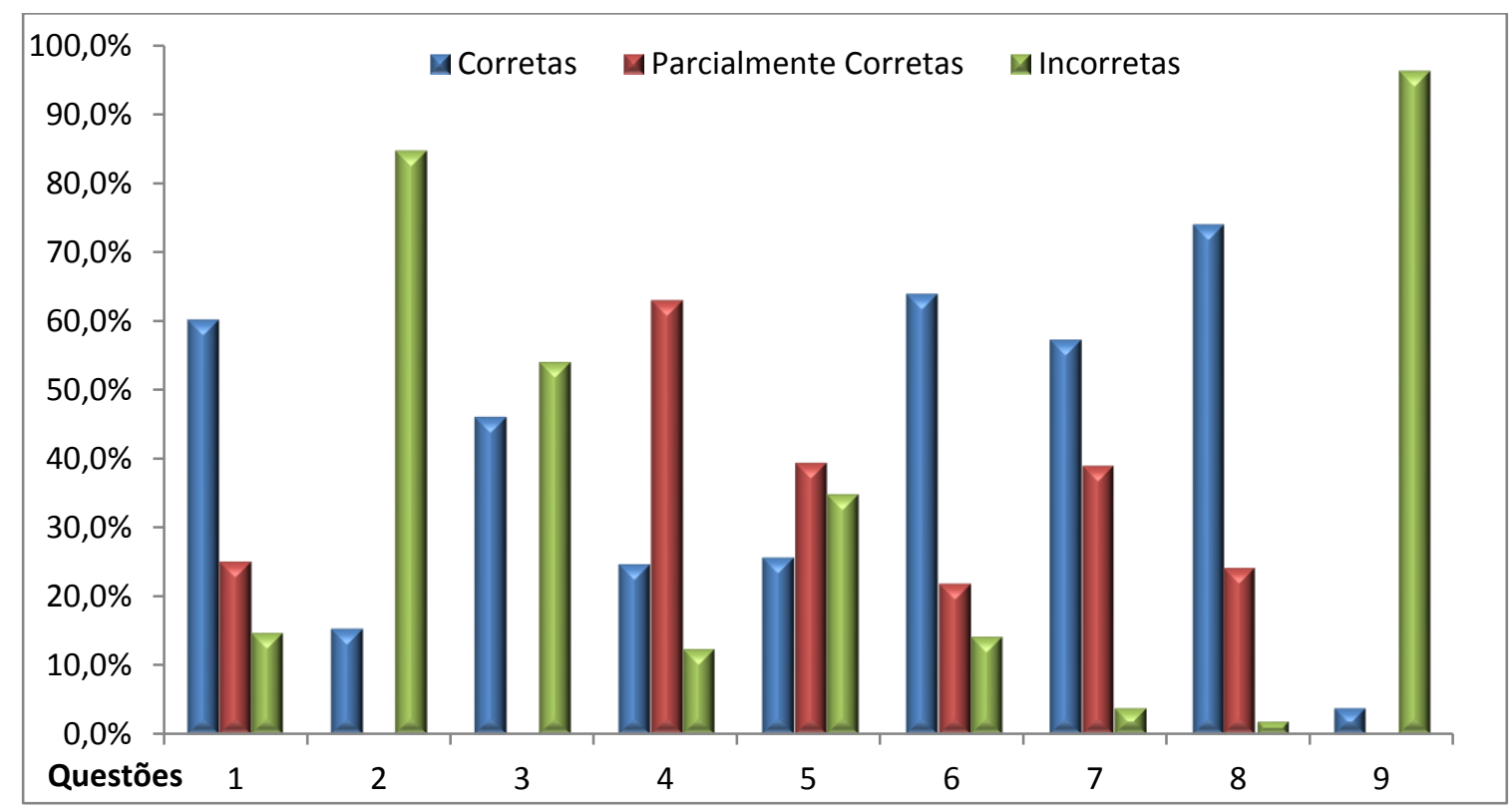

A primeira questão abordava as funções dos macronutrientes, sendo que 98 participantes responderam corretamente $(60,12 \%)$, seguidos das questões consideradas parcialmente corretas com $25,15 \%$. 
A questão número dois tratava do carboidrato em relação à gordura. A maioria $(n=138)$ respondeu essa questão de forma incorreta, totalizando $84,66 \%$ das respostas erradas, tornando-a a segunda questão com maior percentual de erros.

Já na questão de número três, houve divergência das opiniões, com 46,01\% dos participantes tendo afirmado que vitaminas e minerais fornecem calorias ao organismo e 53,99\% respondendo de forma correta, afirmando que não há o fornecimento.

A questão de número quatro continha a identificação de fontes de carboidrato e foram 162 respostas. Do total, 62,96\% responderam à pergunta de forma parcialmente correta, sendo que a maior parte dos participantes $(n=49)$ assinalaram sete opções corretamente (7/10). Os alimentos que mais obtiveram erros foram o mel $(75,93 \%)$ e a pera $(84,57 \%)$, mostrando que os participantes não os consideram carboidratos.

A quinta questão tratava de identificação de nutrientes versus alimentos ( $\mathrm{n}$ $=152$ ), em que a maioria assinalou cinco sugestões de forma correta $(23,68 \%)$. Foram $39,47 \%$ das respostas consideradas como parcialmente corretas, seguido de $34,87 \%$ de respostas incorretas. O nutriente gordura foi o menos assinalado, com $71,42 \%$ de erros.

A questão número seis relacionava alimentos que são substituíveis entre si e 155 indivíduos responderam a essa pergunta, sendo que 99 praticantes o fizeram corretamente $(63,87 \%)$.

Na questão de número sete, encontravam-se alimentos fontes de proteínas, no qual $57,23 \%$ dos participantes que responderam $(n=159)$ souberam indicar os alimentos proteicos (7 ou 8 acertos/8) e 38,99\% acertaram parcialmente a questão. Houve receio em relação ao iogurte, que não foi assinalado em $68,55 \%$ das respostas.

A questão de número oito era sobre as gorduras e apresentou grande número de acertos $(73,91 \%)$ e apenas $1,86 \%$ de erros de 161 respostas.

Já a questão de número nove trouxe muitos erros com relação à distribuição correta dos nutrientes em uma dieta balanceada ( $n=157$ ). Foram $96,18 \%$ de respostas erradas, sendo que $43,58 \%$ dos praticantes assinalaram a questão que continha a predominância do carboidrato e baixa de gorduras: gorduras (15\%), 


\section{Artigo Original \\ Exercício na Saúde, na doença e no esportivo}

proteínas (25\%) e carboidratos (60\%), seguido de $35,89 \%$ das respostas para a distribuição maior para proteínas e baixa de carboidratos: gorduras (25\%), proteínas $(60 \%)$ e carboidratos (15\%).

Pode-se perceber que as questões em que houve maior número de erros eram questões relacionadas ao valor energético dos nutrientes, bem como aos nutrientes que mais devem ser consumidos na dieta. Porém, obteve-se maior conhecimento em questões sobre função dos macronutrientes, seus alimentos-fonte e suas substituições.

A relação entre o conhecimento sobre nutrição e as variáveis $I M C$, frequência da prática de atividade física, tempo de prática e haver sido orientado por nutricionista pode ser observada na Tabela 1.

Pode-se perceber que em relação ao IMC, o conhecimento nutricional se mostrou moderado em todas as classificações (de baixo peso a obesidade), seguidos do conceito baixo de conhecimento. Não foram encontradas diferenças estatísticas significativas.

Em se tratando da frequência semanal de prática, entre os indivíduos que praticam exercícios físicos menos de três vezes por semana, a maioria apresentou conhecimento baixo. Entre os que praticam entre três e quatro vezes e entre cinco ou mais vezes, o conhecimento foi de nível moderado, porém, a porcentagem foi aumentada para aqueles com frequência semanal de prática maior. Foram encontradas diferenças estatísticas significativas, indicando associação entre o conhecimento e a frequência de prática.

De acordo com o tempo de prática, a maioria apresentou nível moderado de conhecimento, porém, indivíduos que praticam há menos de um ano foram os que apresentaram maior porcentagem entre o conhecimento baixo. Aqueles que praticam entre um e quatro anos indicaram maiores valores de conhecimento moderado e aqueles com prática há mais de quatro anos apresentaram as maiores porcentagens entre 0 alto conhecimento, justificando as diferenças estatísticas significativas encontradas, mostrando que os mesmos se comportam de maneira semelhante. 
Exercício na Saúde, na doença e no esportivo

Tabela 1. Conhecimento sobre nutrição entre praticantes de exercício físico de uma academia e associação com o Índice de Massa Corporal, frequência de atividade física, tempo de prática e orientação de nutricionista. Criciúma - SC.

\section{Conhecimento sobre nutrição}

\begin{tabular}{|c|c|c|c|c|c|c|c|}
\hline \multirow[b]{2}{*}{ IMC } & \multicolumn{2}{|c|}{ Baixo } & \multicolumn{2}{|c|}{ Moderado } & \multicolumn{2}{|c|}{ Alto } & \multirow[t]{2}{*}{$P$} \\
\hline & $\mathbf{n}$ & $\%$ & $\mathbf{n}$ & $\%$ & $\mathbf{n}$ & $\%$ & \\
\hline Baixo peso & 02 & 40 & 03 & 60 & 00 & 0,00 & \multirow{4}{*}{0,797} \\
\hline Eutrofia & 15 & 18 & 58 & 69 & 11 & 13 & \\
\hline Sobrepeso & 12 & 21 & 38 & 67 & 07 & 12 & \\
\hline Obesidade & 02 & 29 & 05 & 71 & 00 & 0,00 & \\
\hline Frequência da prática & $\mathbf{n}$ & $\%$ & $\mathbf{n}$ & $\%$ & $\mathbf{n}$ & $\%$ & \\
\hline Menos de 3 vezes & 07 & 46 & 04 & 27 & 04 & 27 & \multirow{3}{*}{0,008} \\
\hline De 3 a 4 vezes & 16 & 20 & 56 & 71 & 07 & 09 & \\
\hline 5 ou mais vezes & 09 & 15 & 46 & 74 & 07 & 11 & \\
\hline Tempo de Prática & $\mathbf{n}$ & $\%$ & $\mathbf{n}$ & $\%$ & $\mathbf{N}$ & $\%$ & \\
\hline Menos de 1 ano & 24 & 36 & 34 & 52 & 08 & 12 & \multirow{3}{*}{$0,000^{\star}$} \\
\hline Entre 1 e 4 anos & 09 & 20 & 35 & 78 & 01 & 02 & \\
\hline Mais de 4 anos & 03 & 06 & 39 & 76 & 09 & 18 & \\
\hline Nutricionista & $\mathbf{n}$ & $\%$ & $\mathbf{n}$ & $\%$ & $\mathbf{n}$ & $\%$ & \\
\hline Com orientação & 10 & 23 & 30 & 70 & 3 & 07 & \multirow[t]{2}{*}{0,659} \\
\hline Sem orientação & 25 & 21 & 78 & 67 & 14 & 12 & \\
\hline
\end{tabular}

* Teste do Qui-quadrado de Pearson. IC de 95\%, com valor $p<0,05$.

Tanto entre os praticantes que afirmaram ter consultado nutricionista quanto os que afirmaram não terem sido orientados, o conhecimento nutricional foi moderado ( $70 \%$ e $67 \%$ respectivamente).

\section{DISCUSSÃO}

Na presente pesquisa, $59 \%$ dos pesquisados praticavam atividade física há mais de um ano. Resultados semelhantes foram encontrados em praticantes de atividade física em uma academia de ginástica da cidade de Juiz de Fora, MG, onde $61,3 \%$ dos 348 indivíduos matriculados praticavam atividade física há mais de um 


\section{Exercício na Saúde, na doença e no esportivo}

ano e $73,2 \%$ relataram uma frequência de prática de atividade física de três a cinco vezes por semana ${ }^{10}$.

Quando observado o conhecimento sobre nutrição e a relação de tempo de prática física, nos indivíduos que se exercitavam há quatro anos ou mais, houve predominância de conhecimento moderado de nutrição (76\%), resultado semelhante ao encontrado nos indivíduos que praticavam atividade física há menos de um ano (52\%) e entre um e quatro anos (78\%). Os que praticavam atividade física há mais de quatro anos obtiveram as maiores porcentagens entre o alto conhecimento de nutrição (18\%). Estes resultados sugerem que o grupo pesquisado, como um todo, apresentava conhecimento de nutrição sem maiores aprofundamentos, o que pode contribuir para uma conduta alimentar inadequada.

Foram constatadas diferenças estatísticas significativas entre 0 conhecimento nutricional e as variáveis frequência da prática e tempo de prática de exercícios físicos. No entanto, na relação entre conhecimento nutricional e as variáveis IMC e orientação de nutricionista não houve diferença significativa.

Em se tratando da frequência semanal da prática física, $90 \%$ dos pesquisados se exercitam três vezes ou mais por semana. Ressalta-se que, dos indivíduos que praticam exercícios físicos menos de três vezes por semana, a maioria apresentou baixo conhecimento de nutrição (46\%). Entre os que praticavam atividade física entre três e quatro vezes e entre cinco ou mais vezes, predominou conhecimento moderado de nutrição ( $71 \%$ e $74 \%$, respectivamente), porém, a maior porcentagem de alto conhecimento de nutrição ficou para os que praticavam atividade física menos de três vezes na semana (27\%).

Ao analisar o IMC dos participantes da pesquisa, pode-se observar predominância de eutrofia, seguida de obesidade. Na relação entre IMC e conhecimento de nutrição, em todas as classificações de IMC predominou o conhecimento nutricional moderado. Com relação ao IMC, resultados semelhantes são relatados na literatura científica, com predominância de eutrofia e sobrepeso/obesidade em praticantes de atividade física ${ }^{11,12,13}$.

Quanto a orientações recebidas por profissional nutricionista, os resultados do presente estudo mostram que $63 \%$ dos praticantes não tinham recebido orientações nutricionais. Ambos os grupos, que tiveram ou não orientação 


\section{Artigo Original \\ Exercício na Saúde, na doença e no esportivo}

nutricional, apresentaram predominância de conhecimento moderado em nutrição, corroborando com outros estudos 5, 7, 13, 14, 15, 16.

Especificamente nas questões sobre conhecimento nutricional, os resultados demonstraram maiores erros nas respostas que abordavam a relação entre valor energético dos carboidratos e gorduras e a distribuição de macronutrientes na dieta, corroborando com outras pesquisas ${ }^{7,}{ }^{18}$.Os maiores acertos foram relacionados às funções dos macronutrientes, alimentos-fonte e respectivas substituições, resultado semelhante ao relatado em outros estudos ${ }^{17,18}$.

Apesar de indivíduos praticantes de exercícios físicos consumirem mais alimentos, quando comparadas com pessoas sedentárias ${ }^{19}$, devem seguir as mesmas recomendações nutricionais para população saudável em geral, com o objetivo de promover a saúde e ter bom desempenho físico ${ }^{20}$. É fundamental a ingestão adequada de carboidratos complexos e de proteínas de alto valor biológico, dentro dos padrões preconizados para uma dieta saudável. Também é preciso atenção quanto ao adequado consumo de ácidos graxos essenciais, vitaminas, minerais e água ${ }^{19}$. Para tal, é fundamental a correta escolha dos alimentos e seus respectivos nutrientes, o que torna o conhecimento nutricional fundamental.

\section{CONCLUSÃO}

Os resultados encontrados na presente investigação, em decorrência do objetivo estabelecido e das discussões decorrentes, permitem concluir que, de modo geral, os praticantes de atividade física pesquisados possuem conhecimento básico sobre nutrição, sem maior aprofundamento.

Ressalta-se a necessidade de maiores informações sobre nutrição, nos praticantes de atividade física avaliados, bem como de orientações nutricionais adequadas, ministradas por profissional nutricionista capacitado em nutrição esportiva.

\section{REFERÊNCIAS}

1. Tirapeghi J. Nutrição, metabolismo e suplementação na atividade física. São Paulo: Atheneu, 2005. 
2. MC Ardle WD, Katch FI, Katch VI. Nutrição: para o desporto e o exercício. Rio de Janeiro: Guanabara Koogan, 2001.

3. Nabholz TV. Nutrição esportiva: aspectos relacionados à suplementação nutricional. São Paulo: Sarvier, 2007.

4. Braggion GF. Suplementação alimentar na atividade física e no esporte - aspectos legais na conduta do nutricionista. Nutrição profissional 2008;4(17):40-50.

5. Hirschbruch MD, Carvalho JR. Nutrição esportiva: uma visão prática. Barueri: Manole, 2014.

6. Pessi S, Fayh, APT. Avaliação do conhecimento nutricional de atletas profissionais de atletismo e triathlon. Rev Bras Med Esp. 2011;17(4):242-5.

7. Bassit RA, Malverdi MA. Avaliação nutricional de triatletas. Rev Paul Educ Fis. 1998;12(1):42-53.

8. Associação Brasileira de Empresas de Pesquisa. Questionário de status socioeconômico, 2015.

9. World Health Organization Physical Status: the use and interpretation of anthropometry, 1995. [acesso em 25 de marco 2016]. Disponível em: http://apps.who.int/iris/bitstream/10665/37003/1/who_trs_854.pdf.

10. Lopes FG, Mendes II, Binoti, MI. Oliveira NP, Percegoni N. Conhecimento sobre nutrição e consumo de suplementos em academias de Ginástica de juiz de fora, brasil. Rev Bras Med Esp. 2015;21(6): 451-6.

11. Marques NF, Marques AC, Fanti YO, Moura, FA. Consumo alimentar e conhecimento nutricional de praticantes de musculação do município de Itaqui-RS. Rev Bras Nut Esp.2015; 9(52):288-97.

12. Ferreira VR, Bento, APN, Silva, MR. Consumo alimentar, perfil antropométrico e conhecimentos em nutrição de corredores de rua. Rev Bras Med Esp. 2015; 21(6):457-61.

13. De Castro NMG, Dattilo M, Lopes, LC Avaliação do conhecimento nutricional de mulheres fisicamente ativas e sua associação com o estado nutricional. RBCE, 2010;32(1):161-72.

14. Tormen CC, Dias RL, Souza, CG. Avaliação da ingestão alimentar, perfil antropométrico e conhecimento nutricional de corredores de rua de Porto Alegre. Rev Bras Nut Esp. 2012;6 (31):4-11.

15. Nicastro H, Dattilo, M, Santos, TR, Vidigal, H, Padilha, G; Zimberg, IZ; Crispim, CA, Stulbach, TE. Aplicação da escala de conhecimento nutricional em atletas profissionais e amadores de atletismo. Rev Bras Med Esp. 2008;14(13):242-5. 


\section{Artigo Original}

Exercício na Saúde, na doença e no esportivo

16. Moreira FP, Rodrigues, KI. Conhecimento nutricional e suplementação alimentar por praticantes de exercícios físicos. Rev Bras Med Esp. 2014;20(5):370-3.

17. Deolindo SM, Moraes CB Consumo alimentar e conhecimento nutricional de idosos praticantes de hidroginástica. RDS. Série: Ciências da Saúde. 2015;16(1): 101-11.

18. Araújo MPS. Nível de conhecimento nutricional de praticantes de corrida do grupo endorfina assessoria esportiva do município de Araguari. Rev Bras Nut Esp. 2012;6(32):97-104.

19.Fernandez MD, Sainz AG, Garzón MJC. Treinamento físico-desportivo e alimentação: da infância à idade adulta, 2a edição, Porto Alegre: Editora Artmed, 2002.

20. Hernandez AJ, Nahas RM. Modificações dietéticas, reposição hídrica, suplementos alimentares e drogas: comprovação de ação ergogênica e potenciais riscos para a saúde Rev Bras Med Esp. 2009;15(3):3-12. 